\title{
Las clasificaciones francesas de los trastornos mentales: Correlatos y diferencias con la CIE-OMS
}

\author{
Rebeca Robles García
}

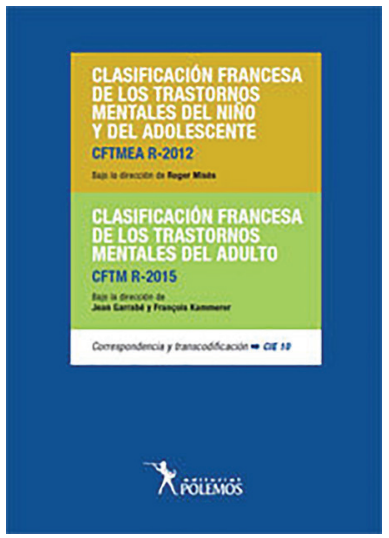

Clasificación francesa de los trastornos mentales del niño y del adolescente (CFMEA R-2012). Clasificación francesa de los trastornos mentales del adulto (CFTM R-2015). 1ra. Edición revisada. Buenos Aires: Polemos, 2017.

DOI: $10.17711 /$ SM.0185-3325.2018.021

\section{Introducción}

El libro que nos ocupa contiene la traducción al español de dos textos de la Asociación Franco-Argentina de Psiquiatría y Salud Mental; uno de ellos presenta la más reciente versión de la clasificación francesa de los trastornos mentales del niño y del adolescente, la CFMEA R-2012, una actualización de su primera edición en el año 2000 (Beraudi-Luppi, 2004); el otro, la actual clasificación francesa de los trastornos mentales de adultos, la CFTM R-2015. En ambos casos se identifican sus correspondencias y transcodificación con la décima versión de la Clasificación Internacional de Enfermedades y condiciones relacionadas con la salud (CIE-10) de la Organización Mundial de la Salud (1989); básica, pero no exclusivamente, con su capítulo $\mathrm{V}$ dedicado a los trastornos mentales y del comportamiento.

\section{Desarrollo y organización de las clasificaciones francesas de los trastornos mentales}

Tanto la CFMEA R-2012 como la CFTM R-2015 fueron desarrolladas por un grupo de expertos franceses con base en el enfoque psicodinámico que incorporaron factores orgánicos y del entorno relacionados con la precipitación y/o mantenimiento o agravamiento de los problemas de la salud mental a lo largo del ciclo vital. Así, a semejanza de las nosologías psiquiátricas más utilizadas a la fecha, las francesas se originan también en el consenso de profesionales destacados en el área y toman en cuenta al menos dos ejes o áreas diagnósticas: el trastorno principal y la presencia de eventos vitales estresantes del entorno. Empero, a diferencia de la CIE (o el DSM de la Asociación Psiquiátrica Americana) destaca que no se trata de una clasificación ateórica.

Esto es notorio desde la organización misma de las categorías denominadas "de base" que se incluyen en su "Eje I General", pues refleja la intención explícita de jerarquizar el funcionamiento mental desde la perspectiva psicodinámica. En el caso de los trastornos mentales de la infancia y la adolescencia, se inicia con los trastornos invasivos del desarrollo, junto con las psicosis, para pasar después a los trastornos neuróticos, las patologías límite y los trastornos reactivos. En el caso de la clasificación de trastornos mentales de los adultos, esta dimensión estructural implica la organización de los grupos diagnósticos en categorías clínicas de base y complementarias. Las de base inician también con los trastornos psicóticos, tímicos y autísticos (categoría 1), para proceder con los neuróticos (categoría 2), los de la organización de la personalidad (categoría 3), los traumáticos y postraumáticos (categoría 4) y las condiciones que no permiten la codificación en las categorías anteriores (categoría 0) pero permiten clasificar provisoriamente un caso a la espera de poder plantear un diagnóstico de funcionamiento como, por ejemplo, frente a un síndrome de Diógenes que todavía no da pruebas de su dimensión de organización compulsiva o de desorganización psicótica (Allilaire, 2017). 


\section{Categorías diagnósticas y ejes de clasificación: Correspondencias CIE-10}

La base psicodinámica de la clasificación se hace también evidente en las categorías diagnósticas específicas y en sus definiciones. Así, por ejemplo, entre las psicosis afectivas del niño (categoría CFMEA R-2012 1.40: Psicosis distímicas del niño) "que pueden aparecer desde los tres o cuatro años en forma de expresiones sintomáticas pertenecientes a la depresión y a la excitación" (pp. 36), se incluyen: "las psicosis afectivas y los trastornos tímicos de estructura psicótica", cuya correspondencia CIE-10 se establece con la categoría F84.8: otros trastornos generalizados del desarrollo + F34.0 Ciclotimia. Y para el caso de los trastornos tímicos del adolescente (categoría CFMEA R-2012 1.41), aunque se reconoce que "no pertenecen estrictamente al registro psicótico, se clasifica en esta sección aquellos cuya severidad y masividad trastornan, temporalmente, el juicio de realidad" (pp. 37), excluyendo "los estados depresivos que sobrevienen de un contexto neurótico, de patología de la personalidad o de trastornos reactivos" (pp. 37); por lo que queda en correspondencia de codificación CIE-10 con la categoría F25.9: Trastornos esquizoafectivos no especificados.

Por otra parte, en ambas clasificaciones francesas, las categorías diagnósticas clínicas de base, tanto principales como complementarias, se incluyen en un EJE I, y se da cuenta entonces de los "factores anteriores o asociados, eventualmente etiológicos" (pp. 167) en un EJE II, que reconoce el papel tanto de factores biológicos como medioambientales en la precipitación y mantenimiento de los trastornos mentales.

Así, por ejemplo, para el caso de la clasificación de trastornos mentales en adultos, se le divide en seis secciones: 1) Factores orgánicos de la infancia y de la adolescencia, 2) Factores y condiciones ambientales de la infancia, la adolescencia y la edad adulta (como carencias afectivas, educativas, sociales y culturales (IA 22), o maltrato y negligencia graves (IA 23), 3) Situaciones relacionadas con el embarazo o con trastornos de la función reproductiva en la edad adulta, 4) Situaciones relacionadas con problemas judiciales o legales en la edad adulta (como tratamiento durante el curso de una encarcelación o medidas de protección judiciales o tutelas), 5) Factores iatrogénicos causantes de trastornos mentales en la edad adulta (incluyendo los imputables a tratamiento o decisión médica no psiquiátrica o por tratamiento psiquiátrico) y 6) Factores orgánicos en la edad adulta.

La transcodificación de estos factores con la CIE-10 se hace con diferentes capítulos de la misma. De este modo, si se trata de un código CFTM R-2015 11.00: Rubéola (pp. 199), la codificación correspondiente CIE-10 será con su código P35.0; y muchos de los problemas sociales advertidos en varios de los demás grupos de factores serán clasificados como códigos Z, por ejemplo, el código CFTM
R-2015 23 (IA Maltrato y negligencia graves) será transcodificado a CIE-11 como Z61.6.

En la CIE-10, además del capítulo $\mathrm{V}$ de trastornos mentales y del comportamiento, existe otro capítulo sobre factores que influyen en el estado de salud o la necesidad de contacto con los servicios de salud, referidos generalmente como códigos Z (en la versión anterior CIE-9 se denominaban códigos V). En este capítulo se incluye una gran cantidad de situaciones vitales estrechamente relacionadas con los avatares de la vida cotidiana que ocasionan discapacidad y malestar importantes (Lewinson, Shankman, Gau, \& Klein, 2004), por lo que son empleados de manera habitual para clasificar las razones de consulta a profesionales de la salud mental (Lobo, González, \& Salvanés, 2006).

Cabe resaltar que, en tanto que no constituyen trastornos mentales en sí mismos, existe controversia en cuanto a la adecuación de su tratamiento (sobre todo de corte farmacológico), sea porque carece de evidencia científica (Helmchen \& Linden, 2000), o porque puede ser ética (Charlton \& McKenzie, 2004) y pragmáticamente cuestionable (Hart, 1971). Quizá sea necesario evaluar rigurosamente la pertinencia y beneficios del tratamiento psicoterapéutico fundamentado en la teoría psicodinámica para aquellos a quienes se concibe que enfrenten una condición equivalente a algún código Z de la CIE-11.

\section{Los diagnósticos no especificados y la necesidad de una CIE con mayor utilidad clínica}

Otra característica sobresaliente de la transcodificación CIE-10 de los diagnósticos propuestos en las clasificaciones francesas que presenta el texto es la abundante correspondencia con categorías no especificadas. Esto puede estar relacionado también con la patologización de malestares de la vida cotidiana que inducen fácilmente a un error tipo 1 (Hernández, Carmona, Franco, \& Escalante, 2006); pero también puede ser el indicativo de la necesidad de una CIE con mayor utilidad clínica en tanto permita identificar adecuadamente casos en necesidad de tratamiento en diferentes culturas y escenarios de atención.

De hecho, ese ha sido el objetivo central que guía el desarrollo de la CIE-11, incluyendo su capítulo de trastornos mentales y del comportamiento (International Advisory Group for the Revision of the ICD-10 Mental and Behavioural Disorders, 2011). Deseablemente, esta nueva versión de la clasificación internacional de la OMS habrá de reducir la frecuencia de uso de categorías no especificadas por los profesionales de la salud mental y las clasificaciones locales o regionales derivadas o transcodificadas de la misma.

\section{Conclusiones}

Con todo y su marcada diferenciación con el resto de los sistemas nosológicos vigentes a la fecha, el libro Clasifi- 
cación francesa de los trastornos mentales del niño y del adolescente (CFMEA R-2012). Clasificación francesa de los trastornos mentales del adulto (CFTM R-2015) es una publicación relevante para los especialistas encargados del diagnóstico y tratamiento de los problemas de salud mental desde la perspectiva psicodinámica que puede ser bien utilizado para el entrenamiento de estudiantes en esta área del conocimiento.

Además, la inclusión de la correspondencia específica de cada categoría nosológica con los diagnósticos de la CIE de la OMS hace posible una suerte de armonización a favor de la integración de un lenguaje profesional. Una taxonomía común no es una tarea menor; no sólo permite cumplir con la obligación de los Estados miembros de la OMS en relación con el reporte estadístico de los problemas de salud mental de un pueblo, facilita también la comunicación $-\mathrm{y}$ comprensión- entre profesionales e investigadores, y esto, y a su vez, la generación y traslación de conocimiento sobre la compleja y dinámica causalidad de los trastornos mentales. Así pues, deseablemente tendrá a la larga cierto valor heurístico en la mejora de los tratamientos interdisciplinarios para quienes tanto sufren a consecuencia de un problema de salud mental. Que así sea, enhorabuena.

\section{REFERENCIAS}

Allilaire, J. F. (2017). ¿Qué lugar ocupa en la nosología psiquiátrica mundial la clasificación francesa CFTM R-2015?. In J. Garrabé \& F. Kammerer (Eds.),
Clasificación francesa de los trastornos mentales del niño y del adolescente (CFMEA R-2012). Clasificación francesa de los trastornos mentales del adulto (CFTM R-2015). Buenos Aires: Polemos.

Charlton, B. G. \& McKenzie, K. (2004). Treating unhappiness: society needs palliative psychopharmacology. British Journal of Psychiatry, 185(3), 194-195.

Organización Mundial de la Salud. (1989). Clasificación Estadística Internacional de Enfermedades y problemas relacionados con la salud. Ginebra: Organización Mundial de la Salud.

Beraudi-Luppi, A. (2004). Clasificación francesa de los trastornos mentales del niño $y$ del adolescente: CFTMEA-R-2000. Buenos Aires: Asociación Franco Argentina de Salud Mental-Polemos.

Hart, J. T. (1971). The inverse care law. The Lancet, 297(7696), 405-412.

Helmchen, H. \& Linden, M. (2000). Subthreshold disorders in psychiatry: clinical reality, methodological artifact and the double-threshold problem. Comprehensive Psychiatry, 41(2), 1-7.

Hernández, J. G. E., Carmona, F. M., Franco, E. D., \& Escalante, A. (2006). Estudio descriptivo de la población psiquiátrica del Instituto Nacional de Perinatología. Ginecología y Obstetricia de México, 74(09), 476-482.

International Advisory Group for the Revision of the ICD-10 Mental and Behavioural Disorders. (2011). A conceptual framework for the revision of the ICD-10 classification of mental and behavioural disorders. World Psychiatry, 10(2), 86-92.

Lewinson, P. M., Shankman, S. A., Gau, J. M., \& Klein, D. N. (2004). The prevalence and comorbidity of subthreshold psychiatric conditions. Psychological Medicine, 34(4), 613-622.

Lobo, A. O., González, R. G., \& Salvanés, F. R. (2006). La derivación a salud mental de pacientes sin un trastorno psíquico diagnosticable. Atención primaria, 38(10), 563-569. 